\title{
The Coreidae of Honduras (Hemiptera: Coreidae)
}

\author{
Carlos A Linares $\ddagger$, Jesus Orozco $\ddagger$ \\ ‡ Insect Collection, Zamorano University, Zamorano, Honduras
}

Corresponding author: Jesus Orozco (jorozco@zamorano.edu)

Academic editor: Laurence Livermore

Received: 04 Apr 2017 | Accepted: 02 Jun 2017 | Published: 05 Jun 2017

Citation: Linares C, Orozco J (2017) The Coreidae of Honduras (Hemiptera: Coreidae). Biodiversity Data Journal 5: e13067. https://doi.org/10.3897/BDJ.5.e13067

\section{Abstract}

\section{Background}

Coreidae bugs are mostly sap-sucking insects feeding on a variety of plants. Despite their abundance and economic importance in Honduras there is little information on the species, their distribution and affected crops. Since knowledge of pest species allows for better management of crops, we aimed to document the diversity of this economically important group. Specimens from four entomological collections in Honduras were studied and an exhaustive search of all available literature was conducted.

\section{New information}

A total of 2,036 insects were examined. The fauna of Honduran coreids is now composed of 68 species. Nineteen species are recorded for the country for the first time and 17 species were found only in literature. Little is known about the biology and economic importance of most of the species.

\section{Keywords}

Taxonomy, diversity, agriculture, pest, Central America. 


\section{Introduction}

Bugs of the Coreidae family are primarily phytophagous insects that feed on plants sucking sap from branches, leaves, flowers and fruits. Many coreids are known pests of ornamentals and crops that can, at times, cause serious damage or even total loss (Henry 2009). Due to their feeding habits, they can cause malformations, rotting, discoloration, and also abortion of the fruit and poor formation of the seed (Mitchell 2000).

Honduras' economy is largely depending on agriculture. Given that pest control depends heavily on proper species identification and management, knowledge of the species becomes paramount for the economy. Despite this, little information on insects from the country is available.

Evans and Halbert (2007) found 26 new aphids in Honduras out of the 46 species known for the country. In Coleoptera, Turnbow et al. (2003b), found that of the 626 species of Honduran cerambycids, 364 were new country records. Similarly, Turnbow et al. (2003a), found 78 bruchids new to the country of the 148 known species. We estimate that at least half of the species of insects in Honduras are known unknowns: species already described that are not recorded for the country. This, together with the relative lack of in-country taxonomists poses a difficulty for, among other things, crop management. It is impossible to know the pest status of an unknown insect.

This work is an effort to provide comprehensive information on the coreid species from Honduras, including their geographic distribution by department and the plants they are known to feed on.

\section{Materials and methods}

Specimens from the following entomological collections in Honduras were examined:

- Colección Entomológica del Centro Universitario Regional del Litoral Atlántico, Ceiba (CURLA).

- Colección Entomológica de la Escuela Nacional de Ciencias Forestales, Siguatepeque (CEEF).

- Museo Entomológico de la Universidad Nacional Autónoma de Honduras, Tegucigalpa (UNAH).

- Zamorano Insect Collection, Zamorano University, Zamorano (EAPZ).

The material was identified by comparison with a reference collection at EAPZ and by using available keys. Label information containing host, date of collection, and distribution was recorded. Additional species information was gathered from the literature.

Geographic and temporal distribution of adults in Honduras as well as biological infomation for all species was obtained from label data. For the new country records the known distribution, outside Honduras, according to the available literature is included. 


\section{List of Honduran Coreids}

\section{Acanthocephala alata (Burmeister, 1835)}

Distribution: Atlántida, Comayagua, Francisco Morazán, and Yoro.

Notes: Specimens examined: 115 (CEEF, CURLA, EAPZ, UNAH).

Temporal distribution: May-September.

Hosts: Phaseolus vulgaris L. (beans) (EAPZ); Annona muricata L. (soursop) (Hernández and Pinzón 2015), and Jatropha curcas L. (Barbados nut) (Grimm and Maes 1997).

Attacks the fruits of Annona muricata L. causing their fall (Hernández and Pinzón 2015).

\section{Acanthocephala declivis (Say, 1832)}

Distribution: Atlántida, Comayagua, and Francisco Morazán.

Notes: Specimens examined: 13 (CEEF, CURLA, EAPZ, UNAH).

Temporal distribution: May-July.

Hosts: Baccharis halimifolia L. (groundsel bush), Baccharis neglecta Britton, and Persea borbonia (L.) Spreng. (redbay) (McPherson et al. 2011).

\section{Acanthocephala femorata (F., 1775)}

Distribution: Atlántida, Comayagua, El Paraíso, Francisco Morazán, and Valle.

Notes: Specimens examined: 108 (CEEF, CURLA, EAPZ, UNAH).

Temporal distribution: January (Passoa 1983), May-July, October.

Hosts: Ipomoea batatas (L.) Lam. (sweet potato), Citrus sinensis (L.) Osbeck (orange), Cucurbita pepo L. (pumpkin) (EAPZ); Solanum tuberosum L. (potato) (Passoa 1983); Helianthus annuus L. (sunflower), Cirsium texanum Buckley, Ambrosia trifida L. (wild hemp), Baccharis neglecta Britton, Chenopodium album L. (pigweed), Cirsium horridulum Michx. (yellow thistle), Ratibida columnifera (Nutt.) Wooton \& Standl. (prairie coneflower), Sorghum halepense (L.) Pers. (Johnson grass), Erigeron quercifolius Lam., Abelmoschus esculentus (L.) Moench (gumbo), Parthenium sp., Gossypium hirsutum L. (cotton), Prunus persica (L.) Batsch (peach), Sorghastrum nutans (L.) Nash (McPherson et al. 2011), and Jatropha curcas L. (Barbados nut) (Alonso and Lezcano 2014). 
Acanthocephala femorata is parasitized by Trichopoda pennipes (F.), (Diptera: Tachinidae). McPherson et al. (2011) recorded Arilus cristatus L. (Hemiptera: Reduviidae), Gryon floridanum Ashmead (Hymenoptera: Scelionidae), and Bicyrtes quadrifasciata Say (Hymenoptera: Crabronidae) as natural enemies.

\section{Anasa bellator (F., 1787)}

Distribution: Unknown in Honduras (Maes and Goellner-Scheiding 1993).

Notes: Temporal distribution: July-October (Maes and Goellner-Scheiding 1993).

Hosts: Tournefortia sp., Zea mays L. (corn), Coffea arabica L. (coffee) (Maes and Goellner-Scheiding 1993), and Myristica fragrans Houtt. (nutmeg) (Brailovsky 1985).

\section{Anasa capaneodes Stål, 1862}

Distribution: Francisco Morazán.

Notes: Specimens examined: 5 (EAPZ).

Temporal distribution: May-July.

Hosts: Tillandsia bourgaei Baker, Tillandsia intumescens L. B. Sm. and Tillandsia prodigiosa (Lem.) Baker (Maes and Goellner-Scheiding 1993).

\section{Anasa linnavuorii Brailovsky, 2016}

Distribution: Yoro (Brailovsky 2016)

Notes: Temporal distribution: June (Brailovsky 2016).

Hosts: Unknown (Brailovsky 2016).

\section{Anasa maculipes Stål, 1862}

Distribution: Olancho (Brailovsky 1985).

Notes: Temporal distribution: Unknown.

Hosts: Cucurbita pepo L. (pumpkin), Quercus sp., Buddleja americana L., and Ageratina adenophora (Spreng.) R.M. King \& H. Rob. (crofton-weed) (Brailovsky 1985).

Anasa scorbutica (F., 1775)

Distribution: Atlántida, Choluteca, Comayagua, El Paraíso, Francisco Morazán, La Paz, Olancho, and Yoro. 
Notes: Specimens examined: 35 (CEEF, CURLA, EAPZ, UNAH).

Temporal distribution: May-December.

Hosts: Cucurbita argyrosperma K. Koch, Zea mays L. (corn), Cucurbita pepo L. (pumpkin) (Passoa 1983); Solanum lycopersicum L. (tomato), Lagenaria siceraria (Molina) Standl. (bottle gourd) (Brailovsky 1985), and Jatropha curcas L. (Barbados nut) (Alonso and Lezcano 2014).

Brailovsky (1985) recorded Ooencyrtus submetallicus Howard (Hymenoptera: Encyrtidae), Anastatus diversus Gahan (Hymenoptera: Eupelmidae), and Gryon carinatifronsuede Say (Hymenoptera: Crabronidae) as natural enemies.

\section{Anasa trilineata Stål, 1870}

Distribution: El Paraíso and Francisco Morazán.

\section{Notes: NEW COUNTRY RECORD}

Specimens examined: 11 (EAPZ).

Temporal distribution: May-July.

Hosts: Sechium edule (Jacq.) Sw. and Zea mays L. (corn) (EAPZ).

Known distribution: Brazil, Colombia, Costa Rica, Ecuador, Nicaragua, Paraguay, Peru, and Venezuela (Packauskas 2010).

\section{Anasa tristis (De Geer, 1773)}

Distribution: Francisco Morazán.

Notes: Specimens examined: 6 (CEEF, EAPZ, UNAH).

Temporal distribution: May-July.

Hosts: Cucurbita argyrosperma K. Koch, Cucurbita pepo L. (pumpkin), Citrullus vulgaris Schrad. (watermelon), and Cucumis melo L. (sweet melon) (Alston and Barnhill 2008).

In the United States this species is considered as one of the important pests of pumpkin and squash. It causes necrosis on the leaves, scars on the fruits and a rapid wilting of the plant. It is parasitized by Trichopoda pennipes (F.) (Diptera: Tachinidae) (Alston and Barnhill 2008).

\section{Anasa uhleri Stål, 1868}

Distribution: Cortés (Brailovsky 1985). 
Notes: Temporal distribution: Unknown.

Hosts: Cucurbita pepo L. (pumpkin), Opuntia streptacantha Lem. and Ageratina adenophora (Spreng.) R.M. King \& H. Rob. (Brailovsky 1985).

\section{Anisoscelis affinis Westwood, 1840}

Distribution: Atlántida, Francisco Morazán, and Olancho.

Notes: Specimens examined: 40 (CEEF, CURLA, EAPZ, UNAH).

Temporal distribution: May-November.

Hosts: Passiflora edulis Sims (passion fruit) (EAPZ), Passiflora quadrangularis L. (badea) (Lerma et al. 1986), and Solanum betaceum Cavanilles (tree tomato) (Lucas et al. 2010).

It is considered as one of the main pests of tree tomato in Ecuador (Lucas et al. 2010).

\section{Camptischium clavipes (F., 1803)}

Distribution: Atlántida.

\section{Notes: NEW COUNTRY RECORD}

Specimens examined: 8 (EAPZ).

Temporal distribution: February.

Hosts: Solanum melongena L. (eggplant) (King and Saunders 1984).

Known distribution: Argentina, Bolivia, Brazil, Guyana, Lesser Antilles, Panama, and Uruguay (Packauskas 2010).

This species sucks sap from tender shoots and fruits on eggplant plantations. It can cause decay and deformation to fruits (King and Saunders 1984).

\section{Catorhintha apicalis (Dallas, 1852)}

Distribution: Distribution in Honduras unknown (Packauskas 2010).

Notes: Temporal distribution: June-September (Báez and Cervantes 2014).

Hosts: Mirabilis jalapa L., Nolina parviflora Kunth (Hemsl.), Sphaeralcea sp., and Gossypium sp. (Báez and Cervantes 2014). 


\section{Catorhintha guttula (F., 1794)}

Distribution: Atlántida, Choluteca, Comayagua, and La Paz.

Notes: Specimens examined: 1 (CURLA).

Temporal distribution: July.

Hosts: Phaseolus vulgaris L. (beans), Zea mays L. (corn) (Passoa 1983); Mirabilis jalapa L. (Cervantes et al. 2014); Cirsium sp., Artemisia vulgaris L., Cucurbita pepo L. (pumpkin), Lyonia sp., Crotalaria sp., Glycine max (L.) Merr. (soy), Gossypium hirsutum L. (cotton), Sida sp., Mimosa pudica L. Mirabilis sp. Ricinus sp., Boerhavia sp., Oryza sativa L. (rice), Solanum lycopersicum L. (tomato), Theobroma sp., and Waltheria americana L. (Maes and Goellner-Scheiding 1993).

\section{Catorhintha selector Stål, 1860}

Distribution: Distribution in Honduras unknown (Brailovsky and Garcia 1987).

Notes: Hosts: Gossypium herbaceum L. (cotton), Boerhaavia diffusa L., Coffea arabica L. (coffee), Waltheria sp. (Maes and Goellner-Scheiding 1993), and Mirabilis jalapa L. (Cervantes et al. 2014).

This species is frequently associated with Nyctaginaceae (Cervantes et al. 2014).

\section{Cebrenis danieli Brailovsky, 1995}

Distribution: Comayagua (Brailovsky 1995).

Notes: Temporal distribution: Unknown.

Hosts: Neurolaena lobata (L.) R. Br. ex Cass., Mikania scandens (L.) Willd., and Verbesina sp. (Brailovsky 1995).

\section{Cebreniscella exitiosa (Brailovsky, 1984)}

Distribution: Distribution in Honduras unknown (Packauskas 2010).

Notes: Temporal distribution: Unknown.

Hosts: Unknown.

\section{Cebrenistella caltumae Brailovsky, 2013}

Distribution: Distribution in Honduras unknown (Brailovsky 2013).

Notes: Temporal distribution: Unknown. 
Hosts: Unknown.

\section{Chariesterus moestus Burmeister, 1835}

Distribution: Comayagua and Francisco Morazán.

Notes: Specimens examined: 11 (EAPZ).

Temporal distribution: May, June, July, October, and December.

Hosts: Oryza sativa L. (rice), Asparagus officinalis L. (asparagus) (EAPZ), and Jatropha curcas L. (Barbados nut) (Alonso and Lezcano 2014).

Recorded in Fabaceae (Maes and Goellner-Scheiding 1993).

\section{Chelinidea tabulata (Burmeister, 1835)}

Distribution: Comayagua and Francisco Morazán.

Notes: Specimens examined: 6 (CEEF).

Temporal distribution: May and October.

Hosts: Opuntia pilifera F.A.C. Weber and Opuntia imbricata (Haw.) DC. (Brailovsky et al. 1994).

\section{Cimolus vitticeps Stål, 1862}

Distribution: Distribution in Honduras unknown (Packauskas 2010).

Notes: Temporal distribution: Unknown.

Hosts: Unknown.

\section{Diactor bilineatus (F., 1803)}

Distribution: Atlántida.

\section{Notes: NEW COUNTRY RECORD}

Number of specimens: 2 (CURLA).

Temporal distribution: July.

Hosts: Passiflora edulis Sims (passion fruit) (Oliveira and Frizzas 2014).

Known distribution: Brazil (Packauskas 2010). 
It is considered one of the most important pests of passion fruit in Brazil (Oliveira and Frizzas 2014).

\section{Holhymenia histrio (F., 1803)}

Distribution: Atlántida, Francisco Morazán, and Olancho.

\section{Notes: NEW COUNTRY RECORD}

Specimens examined: 9 (EAPZ)

Temporal distribution: February-July.

Hosts: Passiflora edulis Sims (passion fruit) (EAPZ), Passiflora nitida Kunth, Passiflora coccinea Aublet, Passiflora gibertii Brown, and Passiflora alata Curtis (Baldin and Boiça 1999).

Known distribution: Argentina, Brazil, Colombia, French Guiana, Nicaragua, Paraguay, Suriname, and Uruguay (Packauskas 2010).

This species is considered an important pest of passion fruit in Brazil (Baldin and Boiça 1999).

\section{Hypselonotus fulvus (De Geer, 1773)}

Distribution: Atlántida and La Paz.

Notes: Specimens examined: 5 (CURLA).

Temporal distribution: August-September.

Hosts: Zea mays L. (corn), Phaseolus vulgaris L. (beans) (Passoa 1983), (Maes and Goellner-Scheiding 1993); Ananas comosus L. (pineapple) (Arellano et al. 2015), Psidium guajava L., Persea americana Miller (avocado), and Gossypium hirsutum L. (cotton) (Pires et al. 2013).

\section{Hypselonotus interruptus Hahn, 1833}

Distribution: Atlántida, Choluteca, Comayagua, Cortés, El Paraíso, Francisco Morazán, Gracias a Dios, and Yoro.

Notes: Specimens examined: 102 (CEEF, CURLA, EAPZ, UNAH).

Temporal distribution: February-July.

Hosts: Eupatorium sp., Rubus adenotrichos Schltdl., Gossypium hirsutum L. (cotton), 
Hosts: Litchi chinensis Sonn., Oryza sativa L. (Rice) (Passoa 1983); Ananas comosus L. (pineapple) (Arellano et al. 2015); Citrus limon (L.) Osbeck (lemon) (USDA 2015); Casearia sylvestris Swartz, Campomanesia xanthocarpa (Mart.) O. Berg., Myrciaria rivularis (Cambess) O. Berg, Acacia meanrsii De Wild., Caesalpinia pluviosa DC., and Cupressus macrocarpa Hart. (cypress) (Thum and Costa 1997).

\section{Hypselonotus lineatus Stål, 1862}

Distribution: Atlántida and Francisco Morazán.

Notes: Specimens examined: 9 (CURLA, EAPZ).

Temporal distribution: January, May-July.

Hosts: Annona reticulata L. (custard-apple), Glycine max (L.) Merr. (soy), Sorghum halepense (L.) Pers. (Johnson grass) (EAPZ), and Jatropha curcas L. (Barbados nut) (Alonso and Lezcano 2014).

\section{Hypselonotus punctiventris Stål, 1862}

Distribution: Atlántida, Choluteca, Comayagua, Copán, Francisco Morazán, and Yoro.

Notes: Specimens examined: 119 (CEEF, EAPZ).

Temporal distribution: April-July and September-October.

Hosts: Sesamum indicum L. (sesame), Sorghum halepense (L.) Pers. (Johnson grass) (EAPZ); Ipomoea batatas (L.) Lam. (sweet potato), Zea mays L. (corn), Coffea arabica L. (coffee), Citrus sp. (Passoa 1983), and Cirsium vulgare (Savi) Tenore (Chordas et al. 2011).

\section{Leptoglossus brevirostris Barber, 1862}

Distribution: Olancho.

\section{Notes: NEW COUNTRY RECORD}

Specimens examined: 1 (EAPZ).

Temporal distribution: August.

Hosts: Zea mays L. (corn) (EAPZ) and Phoradendron leucarpum (Raf.) Reveal \& M. C. Johnst. (Whittaker 1984)

Known distribution: Costa Rica, Mexico, and United States (Packauskas 2010). 


\section{Leptoglossus cinctus (Herrich-Schäffer, 1836)}

Distribution: Atlántida, Comayagua, Francisco Morazán, and Yoro.

Notes: Specimens examined: 39 (CEEF, CURLA, EAPZ, UNAH).

Temporal distribution: October-November.

Hosts: Citrus sp., Psidium guajava L. (guava) (EAPZ), Cereus sp., and Opuntia sp. (Maes and Goellner-Scheiding 1993).

\section{Leptoglossus concolor (Walker, 1871)}

Distribution: Atlántida, Comayagua, Francisco Morazán, Gracias a Dios, and Yoro.

Notes: Specimens examined: 17 (EAPZ).

Temporal distribution: May, July, September, and December.

Hosts: Anacardium occidentale L. (cashew), Bixa orellana L., Psidium guajava L., and Litchi chinensis Sonn. (Mitchell 2000).

\section{Leptoglossus crassicornis (Dallas, 1852)}

Distribution: Yoro.

\section{Notes: NEW COUNTRY RECORD}

Specimens examined: 3 (CURLA).

Temporal distribution: August.

Hosts: Harrisia pomanensis (F.A.C. Weber ex K. Schum.) Britton \& Rose, Opuntia anacantha Speg., Opuntia elata Link \& Otto ex Salm-Dyck, Opuntia discolor Britton \& Rose, Opuntia sulphurea Gillies ex Salm-Dyck, Opuntia paraguayensis K. Schum., Opuntia quimilo K. Schum., and Opuntia ficus-indica (L.) Mill. (Coscarón and Pall 2015).

Known distribution: Argentina, Bolivia, Colombia, Paraguay, and Uruguay (Packauskas 2010). This is the first record for Central America of a species previously believed to be restricted to South America. Further research is needed to clarify the status of this species.

\section{Leptoglossus gonagra $(\mathrm{F} ., 1775)$}

Distribution: Choluteca, Comayagua, Francisco Morazán, and Olancho.

Notes: Specimens examined: 40 (CEEF, EAPZ) 
Temporal distribution: January, May-July, September, and October.

Hosts: Luffa cylindrica (L.) M. Roem., Passiflora edulis Sims (Passion fruit), Citrullus lanatus (Thunb.) Matsum. \& Nakai (watermelon) (EAPZ); Momordica charantia L. (bitter melon), Citrus sinensis (L.) Osbeck (oranges), Punica granatum L. (grenade), Mangifera indica L. (mango), Nicotiana tabacum L. (tobacco), Cucumis melo L. (sweet melon), Citrus paradisi Macfadyen (grapefruit) (Mitchell 2000), and Jatropha curcas L. (Barbados nut) (Alonso and Lezcano 2014).

\section{Leptoglossus lineosus (Stål, 1862)}

Distribution: Atlántida and Francisco Morazán.

\section{Notes: NEW COUNTRY RECORD}

Specimens examined: 2 (EAPZ, UNAH).

Temporal distribution: March-April.

Hosts: Cucurbita sp. (Mitchell 2000).

Known distribution: Mexico (Packauskas 2010).

\section{Leptoglossus oppositus (Say, 1832)}

Distribution: Atlántida and Francisco Morazán.

Notes: Specimens examined: 1 (EAPZ).

Temporal distribution: May-July.

Hosts: Pinus sp., Cucumis sativus L. (cucumber) (Mitchell 2000); Helianthus sp., Cucurbita sp., Citrullus lanatus (Thunb.) Matsum. \& Nakai (watermelon), Carya sp., Yucca sp., Gossypium sp., Morus sp., Psidium guajava L., Zea mays L. (corn), Prunus sp., Pyrus sp., Coffea arabica L. (coffee), Datura sp., and Solanum lycopersicum L. (tomato) (Maes and Goellner-Scheiding 1993).

Maes and Goellner-Scheiding (1993) recorded Bicyrtes quadrifasciatus Lepeletier (Hymenoptera: Crabronidae) as a natural enemy in Nicaragua. Adults are known to be parasitized by Trichopoda pennipes (F.) (Diptera: Tachinidae) (Mitchell 2000).

\section{Leptoglossus zonatus (Dallas, 1852)}

Distribution: Atlántida, Choluteca, Comayagua, Copán, Cortés, El Paraíso, Francisco Morazán, Gracias a Dios, Islas de la Bahía, Lempira, Olancho, and Yoro.

Notes: Specimens examined: 195 (CEEF, CURLA, EAPZ, UNAH). 
Temporal distribution: Year long.

Hosts: Luffa cylindrica Miller, Passiflora edulis Sims (passion fruit), Asparagus officinalis L. (asparagus), Citrus sinensis (L.) Osbeck (orange), Solanum lycopersicum L. (tomato), Zea mays L. (corn), Punica granatum L. (grenada), Anacardium occidentale L. (cashew), Psidium guajava L., Oryza sativa L. (rice), Solanum tuberosum L. (potato) (EAPZ); Cucurbita sp., Triadica sebifera (L.) Small (Chinese tallow), Sorghum sp. (sorghum), Schizocarpum reflexum Rose, Chilopsis linearis (Cav.) Sweet, Jatropha curcas L. (Barbados nut), Actinocheita filicina (DC.) F. A. Barkley (Mitchell 2000); Helianthus sp., Crescentia sp., Hylocereus sp., Schizocarpum sp., Cucumis sp., Phaseolus sp., Persea sp., Gossypium sp., Azadirachta indica A. Juss., Musa sp., Sesamum indicum L. (sesame), and Coffea arabica L. (coffee) (Maes and GoellnerScheiding 1993).

Leptoglossus zonatus is one of the most important and abundant pest species of coreids in Honduras. This species is parasitized by wasps of the genera Geyon, Ooencyrtus, Anastatus and Neorileya. Beauveria bassiana and Metarhizium anisopliae have been used successfully as a control (Mitchell 2000).

\section{Leptoscelis quadrisignatus (Distant, 1881)}

Distribution: Distribution in Honduras unknown (Packauskas 2010).

Notes: Temporal distribution: Unknown.

Hosts: Unknown.

\section{Lycambes andicola (Breddin, 1903)}

Distribution: Atlántida, Comayagua, and Francisco Morazán.

Notes: NEW COUNTRY RECORD

Specimens examined: 4 (CEEF, CURLA, EAPZ, UNAH).

Temporal distribution: July-September.

Known distribution: Bolivia (Packauskas 2010).

Machtima mexicana Stål, 1870

Distribution: Francisco Morazán.

Notes: NEW COUNTRY RECORD

Specimens examined: 7 (EAPZ, UNAH). 
Temporal distribution: May, June, and August.

Known distribution: Mexico and Panama (Packauskas 2010).

\section{Madura perfida Stål, 1862}

Distribution: Distribution in Honduras unknown (Packauskas 2010).

Notes: Temporal distribution: Unknown.

Hosts: Zea mays L. (corn) (Gibson and Carrillo 1959).

\section{Melucha phyllocnemis (Burmeister, 1835)}

Distribution: Francisco Morazán.

\section{Notes: NEW COUNTRY RECORD}

Specimens examined: 1 (UNAH).

Temporal distribution: July.

Hosts: Neurolaena lobata (L.) R.Br. ex Cass., Mikania scandens (L.) Willd., and Verbesina sp. (Barcellos et al. 2008).

Known distribution: Bolivia, Brazil, Mexico, Colombia, and Paraguay (Packauskas 2010).

\section{Melucha quadrivittis Stål, 1862}

Distribution: Copán, Francisco Morazán, and Olancho.

Notes: Specimens examined: 3 (UNAH).

Temporal distribution: May, September, and November.

\section{Mozena lineolata (Herrich-Schäffer, 1842)}

Distribution: Atlántida, Francisco Morazán, and Olancho (Brailovsky and Barrera 2014).

Notes: Specimens examined: 128 (CEEF, CURLA, EAPZ, UNAH).

Temporal distribution: May-October.

Hosts: Prosopis sp. and Atriplex sp. 


\section{Mozena lunata (Burmeister, 1835)}

Distribution: Atlántida, Comayagua, Francisco Morazán, and Yoro.

\section{Notes: NEW COUNTRY RECORD}

Specimens examined: 152 (CEEF, CURLA, EAPZ, UNAH).

Temporal distribution: May-November.

Hosts: Malus domestica Borkh., Cassia sp. (EAPZ); Acacia farnesiana L. Willd. (Ward et al. 1977), Acacia amentacea DC., and Prosopis glandulosa Torr. (Brailovsky et al. 1995).

Known distribution: Costa Rica, Guatemala, Mexico, Nicaragua, and United States (Packauskas 2010).

\section{Mozena lurida (Dallas, 1852)}

Distribution: Distribution in Honduras unknown (Packauskas 2010).

Notes: Temporal distribution: Unknown.

Hosts: Unknown.

\section{Mozena lutea (Herrich-Schaeffer, 1840)}

Distribution: Distribution in Honduras unknown (Packauskas 2010).

Notes: Temporal distribution: Unknown.

Hosts: Unknown.

\section{Nematopus lepidus Stål, 1862}

Distribution: Atlántida, Olancho, and Yoro.

\section{Notes: NEW COUNTRY RECORD}

Specimens examined: 11 (EAPZ).

Temporal distribution: March-August.

Hosts: Persea americana Miller (avocado) (EAPZ).

Known distribution: Mexico and Panama (Packauskas 2010). 


\section{Pachylis nervosus Dallas, 1852}

Distribution: Atlántida, Comayagua, El Paraíso, Francisco Morazán, Islas de la Bahía, Olancho, and Yoro.

Notes: Specimens examined: 255 (CEEF, CURLA, EAPZ, UNAH).

Temporal distribution: Year long.

Hosts: Mimosa sp. and Prosopis laevigata (Willd.) M. C. Johnst. (Brailovsky 1995).

\section{Paryphes anceps Horvath, 1913}

Distribution: Cortés (Horvath 1913)

Notes: Temporal distribution: Unknown.

Hosts: Unknown.

\section{Paryphes flavocinctus Stål, 1860}

Distribution: Atlántida, Francisco Morazán, and Yoro.

Notes: Specimens examined: 8 (CURLA, EAPZ)

Temporal distribution: April-July.

Hosts: Citrullus lanatus (Thunb.) Matsum. \& Nakai (watermelon) and Gurania sp. (Gilbert 1991).

Phthia lunata (F., 1787)

Distribution: Atlántida, Comayagua, and Cortés.

\section{Notes: NEW COUNTRY RECORD}

Specimens examined: 5 (EAPZ).

Temporal distribution: June, August, and September.

Hosts: Capsicum annuum L. (sweet pepper) (EAPZ); Citrullus lanatus (Thunb.) Matsum. \& Nakai (watermelon), and Cucurbita sp. (Maes and Goellner-Scheiding 1993).

Known distribution: Argentina, Brazil, Colombia, Costa Rica, Cuba, Mexico, Panama, Puerto Rico, and Suriname (Packauskas 2010).

This species is reported as a vector of Phytomonas sp. (Godoi et al. 2002). 


\section{Peranthus longicornis (Dallas, 1852)}

Distribution: Atlántida.

\section{Notes: NEW COUNTRY RECORD}

Specimens examined: 1 (EAPZ).

Temporal distribution: April.

Known distribution: Brazil (Packauskas 2010).

\section{Phthiacnemia picta (Drury, 1773)}

Distribution: Atlántida, Comayagua, Choluteca, El Paraíso, and Francisco Morazán.

Notes: Specimens examined: 38 (CEEF, CURLA, EAPZ, UNAH).

Temporal distribution: May-July, August, and September.

Hosts: Ipomoea batatas (L.) Lam. (sweet potato), Solanum tuberosum L. (potato), Solanum lycopersicum L. (tomato) (EAPZ); Pisum sp., Phaseolus vulgaris L. (beans), Trifolium sp., Vicia sp., Vigna sp., Stellaria sp., Citrullus lanatus (Thunb.) Matsum. \& Nakai (watermelon), Cucurbita pepo L. (pumpkin), Cucumis sativus L. (cucumber), Momordica sp., Passiflora edulis Sims (passion fruit), Punica granatum L. (grenade), Sorghum sp., Zea mays L. (corn), Oryza sativa L. (rice), Coffea arabica L. (coffee), Sesamum indicum L. (sesame), Helianthus annuus L. (sunflower), and Nicotiana tabacum L. (tobacco) (Maes and Goellner-Scheiding 1993).

This species is considered an important pests of tomato in Brazil (Da Silva et al. 2003).

\section{Piezogaster auriculatus (Stål, 1862)}

Distribution: Atlántida, Comayagua, Francisco Morazán, and Yoro.

Notes: Specimens examined: 54 (CEEF, CURLA, EAPZ, UNAH).

Temporal distribution: May-October.

Hosts: Citrus sinensis (L.) Osbeck (oranges) and Pachyrhizus sp. (Dealy 2000).

\section{Plapigus circumcinctus Stål, 1860}

Distribution: Atlántida, Comayagua, Francisco Morazán, and Yoro.

Notes: Specimens examined: 66 (CEEF, CURLA, EAPZ, UNAH).

Temporal distribution: May-July and October-December. 
Hosts: Coffea arabica L. (coffee) (Maes and Goellner-Scheiding 1993).

\section{Possaniella oblata Brailovsky, 1999}

Distribution: Atlántida.

\section{Notes: NEW COUNTRY RECORD}

Specimens examined: 1 (CURLA).

Temporal distribution: May.

Known distribution: Brazil (Packauskas 2010).

\section{Romoniella perfecta Brailovsky \& Barrera, 2001}

Distribution: Atlántida, Comayagua, Olancho, and Yoro.

Notes: Specimens examined: 9 (CEEF, EAPZ, CURLA).

Temporal distribution: February, October, and November.

\section{Sagotylus confluens (Say, 1832)}

Distribution: Atlántida, Comayagua, Choluteca, Cortés, El Paraíso, Francisco Morazán, Islas de la Bahía, Olancho, and Yoro.

Notes: Specimens examined: 196 (CEEF, CURLA, EAPZ, UNAH).

Temporal distribution: Year long.

Hosts: Oryza sativa L. (rice), Zea mays L. (corn) (Passoa 1983), Canavalia ensiformis (L.) DC. (EAPZ); Ricinus communis L. (Valdés-Rodríguez et al. 2015), and Senna obtusifolia L. H .S. Irwin \& Barneby (Palmer and Pullen 2000).

This species is considered one of the main pests of fig trees in Mexico feeding on terminal shoots and new leaves. Due to its large size, the latex loss is high and its excreta favor the establishment of fungi on the terminal buds causing them to dry (Valdés-Rodríguez et al. 2015).

\section{Salamancaniella alternata (Dallas, 1852)}

Distribution: Atlántida and Comayagua.

Notes: Specimens examined: 7 (CURLA, EAPZ).

Temporal distribution: May, July, August-November. 
Hosts: Jatropha curcas L. (Barbados nut) (Alonso and Lezcano 2014).

Savius jurgiosus (Stål, 1862)

Distribution: Francisco Morazán.

Notes: NEW COUNTRY RECORD

Specimens examined: 2 (EAPZ).

Temporal distribution: October.

Hosts: Baltimora sp., Buddleja sessiliflora Kunth, Prosopis sp., and Ziziphus sp.

Known distribution: Costa Rica, Guatemala, Mexico, and United States (Packauskas 2010).

\section{Sephina limbata Stål, 1862)}

Distribution: Atlántida and Comayagua.

Notes: Specimens examined: 13 (CEEF, CURLA)

Temporal distribution: January-April.

Hosts: Unknown.

\section{Serranoniella amblysa Brailovsky \& Barrera, 2001}

Distribution: Comayagua and Yoro.

\section{Notes: NEW COUNTRY RECORD}

Specimens examined: 22 (CEEF).

Temporal distribution: June, September-December.

Known distribution: Brazil (Packauskas 2010).

\section{Spartocera fusca (Thunberg, 1783)}

Distribution: Comayagua and Yoro.

Notes: Specimens examined: 71 (CEEF, CURLA, EAPZ, UNAH).

Temporal distribution: Year long.

Hosts: Ipomoea batatas (L.) Lam. (sweet potato), Gossypium sp. (cotton), Solanum lycopersicum L. (tomato), Solanum americanum Miller, Physalis peruviana L. (Maes 
and Goellner-Scheiding 1993); Solanum tuberosum L. (potato) (Passoa 1983), Solanum nigrum L. (Mitchell 2000), and Capsicum annum L. (Mitchell 2000).

Notes: Sarcophaga sternodontis Towns (Diptera: Tachinidae) is a known parasite of $S$. fusca in Nicaragua (Maes and Goellner-Scheiding 1993).

\section{Staluptus marginalis (Burmeister, 1835)}

Distribution: Comayagua, Francisco Morazán, and Yoro.

Notes: NEW COUNTRY RECORD

Specimens examined: 39 (CEEF, EAPZ).

Temporal distribution: May, July, September, and October.

Hosts: Sorghum halepense (L.) Pers. (Johnson grass) (EAPZ); and Cajanus cajan (L.) Millsp. (Maes and Goellner-Scheiding 1993).

Known distribution: Guatemala and Mexico (Packauskas 2010).

Thasopsis formidabilis (Distant, 1893)

Distribution: Atlántida, Comayagua, Francisco Morazán, and Santa Bárbara

Notes: NEW COUNTRY RECORD

Specimens examined: 13 (CURLA, EAPZ).

Temporal distribution: February, May, and September.

Known distribution: Costa Rica and Panama (Packauskas 2010).

\section{Thasus acutangulus (Stål, 1859)}

Distribution: Atlántida and Yoro.

Notes: Specimens examined: 2 (CURLA, EAPZ).

Temporal distribution: December.

Hosts: Prosopis velutina Wooton (Torre-Bueno 1945), and Pithecellobium sp. (EAPZ).

Zicca rubricator $(\mathrm{F} ., 1803)$

Distribution: Unknown (Brailovsky and Cadena 1992).

Notes: Temporal distribution: Unknown. 
Hosts: Schaueria calycobractea Hilsenbeck \& D. L. Marshall, Chamissoa altissima (Jacq.) Kunth and Phytolacca rivinoides Kunth \& C. D. Bouché (Brailovsky and Cadena 1992).

\section{Zicca taeniola (Dallas, 1852)}

Distribution: Atlántida, El Paraíso, Francisco Morazán, and Olancho.

Notes: Specimens examined: 32 (CURLA, EAPZ).

Temporal distribution: December.

Hosts: Glycine max (L.) Merr. (soy), Solanum tuberosum L. (potato), Zea mays L. (corn), Sechium edule (Jacq.) Sw., Daucus carota L. (carrot) (EAPZ), and Capsicum annuum L. (Maes and Goellner-Scheiding 1993).

\section{Discussion}

A total of 2,036 specimens were examined. 68 species are now known from Honduras with 19 being new country records (Table 1). Seventeen species were only found recorded in literature.

\section{Table 1.}

New country records of Coreidae for Honduras

\begin{tabular}{|l|l|l|}
\hline Species & Specimens examined & Collection \\
\hline Anasa trilineata Stål & 11 & EAPZ \\
\hline Camptischium clavipes (F.) & 8 & EAPZ \\
\hline Diactor bilineatus (F.) & 2 & CURLA \\
\hline Holhymenia histrio (F.) & 9 & EAPZ \\
\hline Leptoglossus brevirostris Barber & 1 & EAPZ \\
\hline Leptoglossus lineosus (Stål) & 2 & EAPZ, UNAH \\
\hline Leptoglossus crassicornis (Dallas) & 3 & CURLA \\
\hline Lycambes andicola Breddin & 4 & All collections \\
\hline Machtima mexicana Stål & 7 & EAPZ y UNAH \\
\hline Melucha phyllocnemis (Burmeister) & 1 & UNAH \\
\hline Mozena lunata (Burmeister) & 152 & All collections \\
\hline Nematopus lepidus Stål & 11 & EAPZ \\
\hline Peranthus longicornis (Dallas) & 1 & EAPZ \\
\hline Phthia lunata (F.) & 5 & EAPZ \\
\hline Possaniella oblata Brailovsky & 1 & CURLA \\
\hline
\end{tabular}




\begin{tabular}{|l|l|l|}
\hline Savius jurgiosus (Stål) & 2 & EAPZ \\
\hline Serranoniella amblysa Brailovsky \& Barrera & 22 & CEEF \\
\hline Staluptus marginalis (Burmeister) & 39 & EAPZ, CEEF \\
\hline Thasopsis formidabilis (Distant) & 13 & EAPZ, CURLA \\
\hline
\end{tabular}

Many of the species found in Honduras are known only from one department. We suspect this is not a real reflection of the species' distribution but collecting bias. For nine of the species there is no information on where in Honduras they occur.

\section{Acknowledgements}

We thank the curators at each of the institutions: Karla Cantarero (UNAH), Carlos Salgado (CURLA), and Oscar Leverón (CURLA). This research was funded by the Department of Agricultural Science and Production at Zamorano University.

\section{References}

- $\quad$ Alonso O, Lezcano JC (2014) Artrópodos asociados a Jatropha curcas Linnaeus. Funciones y estrategia para su manejo. Past. Forr 37 (1): 3-16.

- $\quad$ Alston DG, Barnhill JV (2008) Squash Bug (Anasa tristis). Utah Pests Fact Sheet ENT 120 (08): 1-6.

- $\quad$ Arellano G, Vergara C, Bello S (2015) Plagas entomológicas y otros artrópodos en el cultivo de la piña (Ananas comosus var. comosus (L.) Merr., Coppens \& Leal) en Chancahamayo y Satipo, departamento de Junín, Perú. Ecol. Apl 14 (2): 175-189. https://doi.org/10.21704/rea.v14i1-2.94

- Báez J, Cervantes L (2014) Estados inmaduros y fenología de Catorhintha apicalis scrutator (Hemiptera: Heteroptera: Coreidae) en Michoacán, México. Rev. Biol. Trop 62 (2): 579-587. https://doi.org/10.15517/rbt.v62i2.11783

- Baldin E, Boiça A (1999) Desenvolvimento de Holhymenia histrio (Fabr.) (Hemiptera: Coreidae) em frutos de cinco genótipos de maracujazeiro (Passiflora spp.). An. Soc. Entomol Bras 28 (3): 421-427. https://doi.org/10.1590/S0301-80591999000300006

- Barcellos A, Schmidt L, Brailovsky H (2008) Abundance and Species Richness of Coreoidea (Hemiptera: Heteroptera) from Parque Estadual do Turvo. Southern Brazil. Neotrop. Entomol 37 (4): 406-412. https://doi.org/10.1590/S1519-566X2008000400008

- Brailovsky H (1985) Revisión del género Anasa Amyot-Serville (Hemiptera, Heteroptera, Coreidae, Coreinae, Coreini). Monog. Inst. Biol. Univ. Auton. Mex 2: 1-226.

- Brailovsky H (1995) Revisión del complejo "Cebrenis" (Hemiptera-HeteropteraCoreidae-Coreinae-Coreini). Publ. Esp. Inst. Biol. Univ. Auton. Mex 15: 1-124.

- Brailovsky H (2013) Description of one new species of Cebrenistella, and key to the known species (Hemiptera: Heteroptera: Coreidae). Dtsch. Entomol. Z 60: 209-212.

- Brailovsky H (2016) A new species of Anasa from Honduras (Hemiptera: Heteroptera: Coreidae). Entomologica Americana 122 (1-2): 31-36. https://doi.org/10.1664/15RA-032 
- Brailovsky H, Barrera E (2014) Revisional notes on the genus Melucha (Hemiptera, Heteroptera, Coreidae). Dtsch. Entomol. Z 61: 15-22. https://doi.org/10.3897/ dez.61.7048

- Brailovsky H, Cadena A (1992) Revisión del género Zicca (Hemiptera- HeteropteraCoreidae-Coreinae-Coreini). Publ. Esp. Inst. Biol. Univ. Auton. Mex 9: 9-101.

- Brailovsky H, Garcia M (1987) Revisión del género Catorhintha Stål (HemipteraHeteroptera-Coreidae-Coreinae-Coreini). Monog. Inst. Biol. Univ. Auton. Mex 4: 1-148.

- Brailovsky H, Barrera E, Mayorga C, Ortega G (1994) Estadios ninfales de los Coreidos del Valle de Tehuacán, Puebla (Hemiptera-Heteroptera) Chelinidea staffilesi, $C$. tabulata y Narnia femorata. An. Inst. Biol. Univ. Auton. Mex 65 (2): 250-258.

- Brailovsky H, Mayorga C, Ortega G, Barrera E (1995) Estadios ninfales de los Coreidos del Valle de Tehuacán, Puebla, México (Hemiptera-Heteroptera) II. Especies asociadas a Huizacheras (Acacia spp.) y Mezquiteras (Prosopis spp.): Mozena lunata, Pachylis hector, Savius jurgiosus y Thasus gigas . An. Inst. Biol. Univ. Auton. Mex 66 (1): 57-80.

- $\quad$ Cervantes L, Báez J, Brailovsky H (2014) Estados inmaduros de coreidos (Hemiptera: Heteroptera: Coreidae: Coreinae: Coreini: Chelinideini) de Baja California, México. Rev. Mex. Biod 85: 741-752. https://doi.org/10.7550/rmb.43261

- Chordas SW, Tumlison R, Robison HW, Kremers J (2011) Twenty Three True Bug State Records for Arkansas, with Two for Ohio, U.S.A. J. Ark. Acad. Sc 65 (1): 153-159.

- Coscarón M, Pall J (2015) The Tribe Anisoscelini (Hemiptera: Heteroptera, Coreidae) in Argentina. Zootaxa 4033 (3): 411-426. https://doi.org/10.11646/zootaxa.4033.3.6

- Da Silva RA, Carvalho GS, Flores PS (2003) Morfologia externa dos adultos de Phthia picta (Drury, 1770) (Hemiptera, Coreidae). Bol. Veg. Plagas 29: 249-253.

- Dealy B (2000) A Revision of the genus Piezogaster Amyot \& Serville, (Heteroptera: Coreidae: Nematopodini) and the description of two new species. Fort Hays State University, Hays

- $\quad$ Evans GA, Halbert SE (2007) A Checklist of the Aphids of Honduras (Hemiptera: Aphididae). Fla. Entomol 80 (3): 518-523. https://doi.org/10.1653/0015-4040(2007)90 [518:ACOTAO]2.0.CO;2

- Gibson WW, Carrillo JL (1959) Lista de insectos en la colección entomológica de la oficina de estudios especiales, SAG. Inst. Nac. Inv. Agr. Fol. Misc 9: 1-254.

- Gilbert LE (1991) Anguria y Gurania (Cucurbitaceae) (Pepinos de Monte). In: Janzen DH (Ed.) Historia natural de Costa Rica. Editorial de la Universidad de Costa Rica, San José.

- Godoi MM, Serrano MG, Teixeira MM, Camargo EP (2002) A PCR-Based Survey on Phytomonas (Euglenozoa: Trypanosomatidae) in Phytophagous Hemipterans of the Amazon Region. J. Eukaryot. Microbiol 49 (4): 275-279. https://doi.org/10.1111/ j.1550-7408.2002.tb00370.x

- $\quad$ Grimm C, Maes JM (1997) Insectos asociados al cultivo de tempate (Jatropha crucas) en el Pacífico de Nicaragua. Rev. Nica. Ent 42: 16-34.

- Henry TJ (2009) Biodiversity of Heteroptera . In: Foottit E, Adler P (Eds) Insect Biodiversity: Science and Society. Wiley- Blackwell, Oxford.

- Hernández LM, Pinzón JM (2015) Primer Reporte de Piezogaster odiosus Stål y Acanthocephala alata Burmeister (Hemiptera: Coreidae) como plagas de Annona muricata L. Southwest. Entomol 40 (3): 665-668. https://doi.org/10.3958/059.040.0323

- Horvath G (1913) Revisio critica generis Paryphes Burm. et affinium. Annales Musei Nationalis Hungarici 11: 344-373. 
- $\quad$ King AB, Saunders JS (1984) The Invertebrate Pests of Annual Food Crops in Central America: A Guide to Their Recognition and Control. Overseas Development Administration, London.

- Lerma L, Rojas IE, Velasco NR, Figueroa F (1986) Guía práctica para la identificación y manejo de las principales plagas en cultivos frutales. Acta Agron 36 (3): 68-74.

- Lucas K, Maggi J, Yagual M (2010) Creación de una empresa de producción, comercialización y exportación de tomate de árbol en el área de Sangolquí, provincia de Pichincha. BSc dissertation. Escuela Politécnica del Litoral, Guayaquil.

- Maes JM, Goellner-Scheiding U (1993) Catálogo de los Coreidae (Heteroptera) de Nicaragua. Rev. Nica. Ent 25: 1-19.

- McPherson JE, Packauskas RJ, Sites RW, Taylor SJ, Bundy CS, Bradshaw JD, Mitchell PL (2011) Review of Acanthocephala (Hemiptera: Heteroptera: Coreidae) of America north of Mexico with a key to species. Zootaxa 2835: 30-40.

- $\quad$ Mitchell PL (2000) Leaf-footed bugs (Coreidae). In: Schaefer CW, Panizzi AR (Eds) Heteroptera of Economic Importance. CRC Press, Boca Raton, FL.

- $\quad$ Oliveira CM, Frizzas MR (2014) Principais Pragas do Maracujazeiro Amarelo (Passiflora edulis f. flavicarpa Degener) e seu Manejo. EMBRAPA Cerr. Doc 323: 1-44.

- Packauskas R (2010) Catalog of the Coreidae, Or Leaf-footed Bugs, of the New World. Fort Hays Studies

- $\quad$ Palmer WA, Pullen KR (2000) The Phytophagous Arthropods Associated with Senna obtusifolia (Caesalpiniaceae) in Mexico and Honduras and Their Prospects for Utilization for Biological Control. Biol. Con 20 (1): 76-83. https://doi.org/10.1006/ bcon.2000.0879

- Passoa S (1983) Lista de los insectos asociados con los granos básicos y otros cultivos selectos en Honduras. Ceiba 25 (1): 7-18.

- $\quad$ Pires EM, Nogueira RM, da Silva CJ, Pelissari F, Ferreira JAM, Soares MA (2013) New Sucking Coreids Species in Psidium guajava. Sci. Elec. Arch 4: 31-35.

- Thum AB, Costa EC (1997) Coreidae (Heteroptera) associados a espécies florestais. Cienc. Flo 7 (1): 27-31.

- Torre-Bueno JR (1945) Random notes on Thasus acutangulus . Bull. Brooklyn Ent. Soc 40: 83.

- Turnbow RH, Cave RD, Kingsolver JM (2003a) An Annotated Checklist of the Bruchidae of Honduras. Ceiba 44 (2): 269-278.

- Turnbow RH, Cave RD, Thomas MC (2003b) A list of the Cerambycidae of Honduras, with additions of previously unrecorded species. Ceiba 44 (1): 1-43.

- $\quad$ USDA (2015) Risk Assessment for the Importation of Fresh Lemon (Citrus limon (L.) Burm. f.) Fruit from Northwest Argentina into the Continental United States. USDA, Raleigh, N.C..

- Valdés-Rodríguez OA, Pérez-Vázquez A, Palacios-Wassenaar OM (2015) Insectos plaga en cultivo asociado de Ricinus communis y Moringa oleifera en el centro de Veracruz, México. Rev. Mex. Cienc. Agr. Pub. Esp 11: 2233-2239.

- Ward CR, O’Brien CW, O’Brien LB, Foster DE, Huddleston EW (1977) Annotated Checklist of New World Insects Associated with Prosopis (Mesquite). U.S. Dep. Agric. Tech. Bull 1557: 1-115.

- Whittaker PL (1984) The insect fauna of mistletoe (Phoradendron tomentosum, Loranthaceae) in southern Texas. Southwest. Nat 29: 435-444. https:// doi.org/10.2307/3670996 